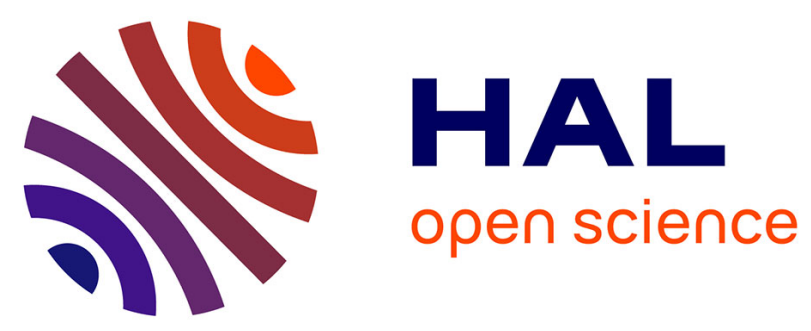

\title{
Design and Fabrication of GaInAsP/InP VCSEL with two a-Si/a-SiNx Bragg reflectors
}

Christophe Levallois, Alain Le Corre, Olivier Dehaese, Hervé Folliot, Cyril Paranthoen, Christophe Labbé, Slimane Loualiche

\section{To cite this version:}

Christophe Levallois, Alain Le Corre, Olivier Dehaese, Hervé Folliot, Cyril Paranthoen, et al.. Design and Fabrication of GaInAsP/InP VCSEL with two a-Si/a-SiNx Bragg reflectors. Optical and Quantum Electronics, 2006, 38 (4-6), pp.281-291. 10.1007/s11082-006-0029-2 . hal-00488026

\section{HAL Id: hal-00488026 https://hal.science/hal-00488026}

Submitted on 1 Jun 2010

HAL is a multi-disciplinary open access archive for the deposit and dissemination of scientific research documents, whether they are published or not. The documents may come from teaching and research institutions in France or abroad, or from public or private research centers.
L'archive ouverte pluridisciplinaire HAL, est destinée au dépôt et à la diffusion de documents scientifiques de niveau recherche, publiés ou non, émanant des établissements d'enseignement et de recherche français ou étrangers, des laboratoires publics ou privés. 


\section{Design and Fabrication of GaInAsP/InP VCSEL with two a-Si/a-SiN ${ }_{x}$ Bragg reflectors}

C. Levallois, A. Le Corre, O. Dehaese, H. Folliot, C. Paranthoen, C. Labbé and S. Loualiche.

Laboratoire d'Etude de Nanostructures Semiconductrices - CNRS UMR FOTON 6082

INSA de Rennes, 20 Avenue des Buttes de Coësmes, 34043 Rennes Cedex, France

Tel: +33223238424

Fax: +33223238618

e-mail: christophe.levallois@ens.insa-rennes.fr

We report on the design and fabrication of a $1.55 \mu \mathrm{m}$ wavelength VCSEL which consists of two dielectric Bragg mirrors and a InGaAsP based active region. The dielectric materials are amorphous silicon and amorphous silicon nitride. Layers of such materials have been deposited by magnetron sputtering and analyzed in order to determine their optical properties. A large refractive index difference of 1.9 is found between these materials. Distributed Bragg reflectors based on these dielectric materials quarter wave layers have been studied by optical measurements and confronted to theoretical calculations based on the transfer matrix method. A maximum reflectivity of $99.5 \%$ at $1.55 \mu \mathrm{m}$ and a large spectral bandwidth of $800 \mathrm{~nm}$ are reached with only four and a half periods of a-Si/a-SiN $\mathrm{N}_{\mathrm{x}}$ The VCSEL was fabricated by metallic bonding process. This method allows to bond an InPbased active region as the gain medium on a Si substrate thanks to the formation of a Au-In alloy. This process is performed at a low temperature of $240^{\circ} \mathrm{C}$ without damaging the optical properties of the microcavity. This VCSEL has been characterized by an optical pumping experiment with a low and a high density optical power and a laser emission has been obtained at room-temperature. 


\section{Introduction}

Vertical cavity surface emitting lasers (VCSELs) emitting at $1.55 \mu \mathrm{m}$ are a promising light sources for low-cost telecommunication systems, especially in emerging metro fiberoptic networks. VCSELs presents interests such as wafer testing before packaging, high coupling efficiency or fabrication in array configuration (A. Karim et al. 2001). However, there are some obstacles to the realization of long-wavelength VCSEL operating in the 1.3$1.55 \mu \mathrm{m}$ range. The lack of lattice-matched high reflective Distributed Bragg Reflectors (DBRs) implies the growth of DBRs with a great number of periods. The largest refractive index that can be obtained on InP-based material need the growth of more than twenty periods of AlGaAsSb/AlAsSb to achieve a high reflectivity at $1.55 \mu \mathrm{m}$ (S. Nakagawa et al. 2001). Another approach consists in using AlAs/GaAs DBR with structures based on InP/InGaAsP active region by using the wafer fusion technique. This method allows to obtain continuouswave $(\mathrm{CW})$ operation thanks to good thermal properties of AlAs and GaAs materials (A. Syrbu et al. 2001). However, high temperatures are necessary to operate wafer-fusion and the fusion bonded interface is located inside the microcavity, close to the active region. Therefore, a good surface preparation is required to ensure a high interface bonding quality. An approach that can be employed consists in using dielectric DBRs, such as $\mathrm{TiO}_{2}-\mathrm{SiO}_{2}$ (D. Sun et al. 2003). This method presents some advantages, like using materials with a high refractive index difference and deposition techniques which are less expensive than epitaxy. In order to integrate two efficient dielectric DBRs with an active region lattice-matched on a InP substrate, a low temperature metallic bonding process can be employed (H.C. Lin et al. 2002; C. Levallois et al. 2005). In this case, the bonding interface is located outside the VCSEL cavity which ensures a good interface quality between the active region and the dielectric Bragg mirrors. 
In the present work, we report on the study of dielectric materials in order to realize an efficient DBR in the $1.5 \mu \mathrm{m}$ region. The dielectric materials used are amorphous silicon (a-Si) and amorphous silicon nitride $\left(\mathrm{a}-\mathrm{SiN}_{\mathrm{x}}\right)$. The a-Si is low cost, highly pure, easy to sputter, and it presents an optical index with a large contrast with a-SiN ${ }_{\mathrm{x}}$. Optical indexes and absorption coefficients of a-Si and $\mathrm{a}-\mathrm{SiN}_{\mathrm{x}}$ have been measured in order to optimize the magnetron sputtering parameters. A Bragg mirror with these materials has been characterized by transmittance and reflectivity measurements. The reflectivity reaches a value of $99.5 \%$ at 1.55 $\mu \mathrm{m}$ for four and a half periods. A VCSEL cavity has been designed with two a-Si/a-SiN Bragg mirrors thanks to a metallic bonding process using a Si substrate and a Au-In alloy. The low temperature used during operation allows to reduce damages on dielectric DBR and InP based devices. This VCSEL has been characterized and laser emission has been obtained at room-temperature (RT).

\section{Dielectric material properties}

Optical material properties need to be known to realize an efficient DBR. The refractive index difference between the two materials determines the number of periods needed to achieve a high reflectivity mirror. Furthermore, VCSEL operation is very sensitive to DBR losses which can mainly originate from residual absorption. These optical properties were characterized by using the transfer matrix method (TMM) (J.B. Pendry and A. MacKinnon 1992) and transmission measurements with a FTIR (Fourier Transform InfraRed) spectrometer.

In order to know these optical properties several bulk layers with a thickness of $1 \mu \mathrm{m}$ have been deposited on glass substrate with various compositions. A magnetron sputtering system with a silicon target was employed to deposit these films. All the sputtered layers were realized on a plate cooled by water circulation. We studied the influence of the $\mathrm{N}_{2}$ flow rate 
on optical index with a total introduced gas pressure $\left(\mathrm{Ar}+\mathrm{N}_{2}\right)$ maintained constant at $10^{-2}$ Torr. As it is reported in Fig. 1, a large optical index variation from 1.83 to 3.74 can be obtained by adjusting this parameter. The value of 3.74 obtained for the a-Si refractive index is higher than for crystalline Si and matches published data values (G.N. Waddell et al. 1984; G.K. Hubler et al. 1979). Consequently, it allows to obtain a large refractive index difference with a-SiN ${ }_{x}$ realized at a high nitrogen flux $(\Delta \mathrm{n} \sim 1.9)$. The decrease of the refractive index with nitrogen is accompanied by a decrease of the absorption from $380 \mathrm{~cm}^{-1}$ to a value close to $20 \mathrm{~cm}^{-1}$ (Fig. 1). Thus, it is possible to realize layer with an intermediate composition which allows to reduce the optical absorption in the layer. However, the refractive index obtained is lower than the one determined previously with a-Si. Thus, the refractive index difference with $\mathrm{a}-\mathrm{SiN}_{\mathrm{x}}$ realized at a high nitrogen flux would be lower and reflectivity simulations shown that it would imply a more pairs for the DBR to achieve the same reflectivity. As it can be seen in Fig.1, the refractive index of a-SiN $\mathrm{x}_{\mathrm{x}}$ is not sensitive to a small variation of the flux for a high $\mathrm{N}_{2}$ flow. With these conditions, a reproducible refractive index is obtained easier than with materials deposited at an intermediate flux value.

Consequently, a-Si with no $\mathrm{N}_{2}$ flux and a-SiN $\mathrm{N}_{\mathrm{x}}$ realized at a high $\mathrm{N}_{2}$ flow rate are the two selected materials to realize the dielectric DBR. Even if a residual absorption is present for a-Si layers, the obtained value of $380 \mathrm{~cm}^{-1}$ is consistent with published values (B.S. Richards et al. 2004), the large refractive index contrast yields a very short penetration depth of the optical mode from the inner cavity into the Bragg mirror. For this reason and because the total thickness of silicon is small in the Bragg mirror, low losses by absorption are expected. This refractive index difference between these two materials is higher than the ones obtained with conventional $\mathrm{SiO}_{2} / \mathrm{TiO}_{2}$ dielectric materials used for VCSEL operate at 1.55 $\mu \mathrm{m}$. Furthermore, thermal conductivities of $\mathrm{SiO}_{2}$ or $\mathrm{TiO}_{2}$ layers are lower than the one reported for a-SiN ${ }_{x}$ layers (J. Piprek et al. 1995). Thus, a-Si/a-SiN $\mathrm{S}_{\mathrm{x}}$ as a bottom mirror is a 
promising candidate for a $\mathrm{CW}$ lasing operation of the VCSEL. In addition, these materials do not need an auxiliary ion bombardment to reach good material quality with high compacity as it is required for oxygen-based dielectric materials such as $\mathrm{SiO}_{2}$.

\section{Dielectric DBR characterization}

A dielectric Bragg mirror with those materials has been designed and characterized. The large refractive index between the two materials led us to deposit only four and half period of a-Si/a-SiN $\mathrm{x}_{\mathrm{x}}$ on a glass substrate to achieve a high reflectivity. The sputtering rates have been studied previously to ensure a DBR with a resonant wavelength centered at 1550 nm. First, transmittance characterization was realized by FTIR measurement using an uncooled Ge detector. As it is illustrated in Fig. 2, the transmittance measurement has been compared with the simulated transmission. A good agreement between calculated and measured spectra is obtained for the whole spectral range. A such result is achieved when dependences about the refractive index and the absorption with wavelength are taking into account for the two materials. This result shows that optical constants, thicknesses, and composition of layers are well controlled and remains constants during sputtering deposition. The minimum transmission is observed at $1550 \mathrm{~nm}$ and for this wavelength, the transmittance is equal to $0.2 \%$.

A reflection measurement was also performed with the same FTIR spectrometer. In this case, this commercial system forced us to work with an incidence angle of $27^{\circ}$. For this measurement, the reflectivity of the Bragg mirror is compared to a gold mirror reflectivity. These measures allows to obtain an evaluation of the experimental reflectivity situated between $99.2 \%$ and $99.6 \%$. This measurement has been compared to a simulated spectrum. Due to the optical incidence angle of $27^{\circ}$ of the spectrometer, the TE and TM polarizations of the incident ray have been taken into account in the TMM. As for transmittance 
measurements, a good agreement between the simulated and measured spectrum is reached, as it is seen in Fig. 3. In order to obtain the $3 \mathrm{~dB}$ spectral bandwidth, simulations were performed above the germanium detector cut off wavelength. A spectral bandwidth larger than $800 \mathrm{~nm}$ is deduced from this simulation. A new reflectivity computation was performed in the case of a normal incidence using experimental material parameters as input values. The simulated reflectivity obtained is greater than $99.5 \%$ at a center wavelength of $1.55 \mu \mathrm{m}$.

The stability of this Bragg mirror at RT has been verified over several months and no variations of the reflectivity has been observed. The DBR mirror is also found to be very stable when it was annealed up to $240^{\circ} \mathrm{C}$ and stored. This stability of reflectivity with time and temperature allowed us to use it with a metal bonding process in order to realize a VCSEL.

\section{VCSEL design and fabrication}

The semiconductor part of the resonator is grown by molecular beam epitaxy (MBE). The active region is formed by four half-wave periods and a quarter-wave period of InP layer. Each half-wave period of the active region contains a lattice-matched group of five InGaAs quantum wells $(\mathrm{QWs})$ and $\operatorname{six} \operatorname{In}_{0.8} \mathrm{Ga}_{0.2} \mathrm{As}_{0.435} \mathrm{P}_{0.565}\left(\mathrm{Q}_{1.18}\right.$ : quaternary alloy emitting at 1.18 $\mu \mathrm{m})$ barriers separated by InP layers. Each QWs group is surrounded by $\mathrm{Q}_{1.18}$ layers of controlled thickness in order to obtain an efficient optical absorption of the pump laser. This design of the active region allows to obtain an homogenous optical absorption of the pump power and then an homogenous carrier injection (Geske et al. 2004). As it is represented by the step 1 in Fig. 4, the realization of the active region is followed by the growth of a semiconductor DBR of four and a half period. The semiconductor materials used are InP and $\mathrm{In}_{0.63} \mathrm{Ga}_{0.37} \mathrm{As}_{0.8} \mathrm{P}_{0.2}$ as low and high refractive index, respectively. The refractive index difference of $\Delta \mathrm{n}=0.27$ (Reddy et al, 2002) between these two materials is too much low to 
produce a Bragg mirror with a sufficient reflectivity. From the inside of the cavity, the calculated reflectivity for such a Bragg mirror is close to $15 \%$. However, this short DBR presents two advantages: In the final structure the a-Si absorption is reduced and the semiconductor material thickness is increased inside the VCSEL cavity which yields to a more mechanical robust structure when it is bonded to a silicon substrate after the removal of the InP substrate. As it is reported in Fig. 4, this step is followed by the deposition of a four a$\mathrm{SiN}_{\mathrm{x}} / \mathrm{a}-\mathrm{Si} \mathrm{DBR}$ rising the reflectivity of the bottom mirror to a theoretical value of $99.4 \%$. On top of this first Bragg mirror, a $50 \mathrm{~nm}$ thick titanium followed by a $500 \mathrm{~nm}$ thick gold layers were deposited by electron-beam evaporation. The same operation is realized on a silicon substrate. The thin titanium layer is used to produce a better adhesion of the gold layer and to prevent the diffusion of gold atoms through the DBR. Above the gold layer, the deposition of an indium layer on the $1 \times 1 \mathrm{~cm}^{2}$ sample area by thermal evaporation is realized. As it is illustrated by step 3 in Fig. 4, the sample and the silicon substrate are joined together. Thus, the indium layer is sandwiched between the two gold layers. A bonding pressure of $5 \mathrm{~kg} . \mathrm{cm}^{-2}$ is applied during the annealing process to ensure intimate contact between the Si substrate and the sample. The annealing is realized in a furnace set at a temperature of $260^{\circ} \mathrm{C}$ in a clean room normal atmosphere. The highest temperature reached by the sample after 20 minutes annealing is $240^{\circ} \mathrm{C}$. According to the Au-In phase diagram, an Au-In metallic alloy can be formed (Lee et al. 2001). Once the bonding between the Si substrate and the dielectric DBR is completed (step 4), the InP substrate is removed prior to the deposition of the top DBR. The InP substrate is first reduced to about $80 \mu \mathrm{m}$ by mechanical polishing. Finally, step 5 shows the remaining InP which is completely removed by a selective chemical etching using a mixture of $\mathrm{HCl}$ and $\mathrm{H}_{3} \mathrm{PO}_{4}$. The etching of InP stops on the InGaAs layer which is removed by an other selective etching solution to expose the active region. To prevent lateral etching of the layers located under InGaAs stop etch layer, epoxy resin is placed around the sample. The 
process ends up with the deposition of the top DBR which consists in five periods a-Si/a-SiN mirror. This Bragg mirror is designed to reach a high reflectivity value of $99.5 \%$ at $1550 \mathrm{~nm}$ and a reflectivity value lower than $30 \%$ at the pump wavelength $(\lambda=1.064 \mu \mathrm{m})$.

\section{VCSEL characterizations}

In order to obtain optical properties of the cavity, the fabricated device has been characterized by reflectivity and photoluminescence measurements. The reflectivity measurement with an incidence angle of $27^{\circ}$ has been compared to the theoretical spectrum, as described previously. Prior metallic bonding process, the thicknesses of QWs and epitaxial Bragg mirror are extracted by $\mathrm{X}$ ray measurements to be used as input values in simulated reflectivity program. Except for the shortest wavelengths, where the experimental reflectivity is lower than the expected one, the simulated and experimental microcavity spectra reported in Fig. 5 are in good agreement. This result proves that the optical properties of the microcavity and the reflectivity of the bottom mirror have not been damaged by the metallic bonding process. The Fig.5 inset shows the reflectivity spectrum for a narrow spectral range around the resonant wavelength centered at $1533 \mathrm{~nm}$. The low decrease of the reflectivity at this wavelength is attributed to the absorption of QWs which is enhanced due to the cavity effect. The absorption of QWs (InGaAs/Q $\left.{ }_{1.18}\right)$ deduced from previous FTIR measurements on special samples have been used in simulation parameters in order to reproduce the reflection dip observed in the Fig.5 inset. For this resonant wavelength, MQW (Multi-QW) absorption is close to $4000 \mathrm{~cm}^{-1}$. This wavelength is surrounded by two others resonant wavelengths for the cavity. One of them is located at $1.38 \mu \mathrm{m}$ and is not clearly observable. The noisy signal for wavelengths close to $1.4 \mu \mathrm{m}$ is due to water absorption. The second one is located to longer wavelengths, at $1.7 \mu \mathrm{m}$. For normal incidence, this cavity mode is shifted to a longer wavelength and simulated reflectivity give a resonant wavelength of $1542 \mathrm{~nm}$ as it can be 
observed in Fig. 6. This resonant wavelength has been also compared with the position of the luminescence peak emitted by the VCSEL. As reported in Fig. 6, we observe a luminescence peak centered at $1543 \mathrm{~nm}$ close to the cavity mode expected by the simulated reflectivity. The photoluminescence characterization of the VCSEL has been realized with a CW YAG laser $(\lambda$ $=1064 \mathrm{~nm}$ ). The pump light with a power of $100 \mathrm{~mW}$ was focused to a spot size of about 500 $\mu \mathrm{m}$ onto the top mirror of the VCSEL and a spectrum analyzer with a resolution of $1 \mathrm{~nm}$ was used. In the same conditions, we performed photoluminescence characterizations of 7 QWs (InGaAs/Q ${ }_{1.18}$ ) equivalent to the ones used in VCSEL cavity. The full width at half maximum (FHWM) of the InGaAs/Q ${ }_{1.18}$ QWs photoluminescence spectrum narrows considerably from $93 \mathrm{~nm}$ to $9 \mathrm{~nm}$ when the QWs are placed inside microcavity. Other luminescence peaks located in the proximity of the main mode of the cavity can be observed. The intensity for these other wavelengths are more than 40 times lower. The positions of these luminescence peaks are in agreement with the other optical modes predicted by the simulated reflectivity. This observation allows to deduce the free spectral range of the cavity which is higher than $130 \mathrm{~nm}$. In order to reach laser emission, a more powerful pump laser was needed. So, the continuous-wave YAG was replaced by a pulsed Q-switched YAG laser at $1064 \mathrm{~nm}$ wavelength. The pump pulse width is $1 \mathrm{~ns}$ with a repetition rate of $6.6 \mathrm{kHz}$. This photopumping experiment consists in focusing the laser beam with a microscope objective $(\times 10)$ on the top mirror of the VCSEL with a spot diameter of $10 \mu \mathrm{m}$. The laser emission is collected back by the same objective and transmitted through a beam splitter. The signal is injected in a large core diameter optical fiber connected to a spectrum analyzer. The average signal emitted by the VCSEL versus the peak power of the pump is reported in Fig. 7. A laser emission has been observed at RT and the laser spectrum for the threshold value is reported as inset in the figure with an emission wavelength of $1535 \mathrm{~nm}$. This wavelength is shorter than the one observed in the photoluminescence measurement. However, the threshold value is 
reached for a $250 \mathrm{~mW}$ pump peak power which is higher than the energy density used in photoluminescence. So, this shift to smaller wavelength can be explained by the large carrier density injected in QWs. This threshold value has not been corrected by the reflection of the top mirror at the pump wavelength. However, this rather high threshold value did not allow the CW operation of the VCSEL, but can be partially explained by an insufficient control of epitaxial layers thicknesses during the growth of the active region. Consequently, the different MQWs are not exactly located at the stationary field anti-nodes when the resonant wavelength is $1.55 \mu \mathrm{m}$ and fine tuning between the resonant wavelength and the maximum optical gain of the QWs is not reached. Nevertheless, this preliminary laser characterization shows that a laser emission can be obtained at RT. Optimizations about the design of the active region will be carried out in the near future to reduce the threshold optical pump power. Other optimizations, like the etching of the dielectric materials to define a mesa structure are under study. A such structure will allow the deposition of intracavity contacts layers in order to obtain an electrically pumped device (H.C. Lin et al. 2002).

\section{Conclusion}

In summary, a-Si/a-SiN $\mathrm{S}_{\mathrm{x}} \mathrm{DBR}$ is an alternative approach to provide high reflectivity mirror for long-wavelength VCSELs. The refractive index difference between these two materials is close to 1.9 at $1.55 \mu \mathrm{m}$, and it requires only four and half a-Si/a-SiN $\mathrm{S}_{\mathrm{x}}$ stack of $\lambda / 4$ thickness to achieve a reflectivity of $99.5 \%$ and a very large spectral bandwidth of $800 \mathrm{~nm}$. The mirror is also found to be stable when stored during several months at RT in air ambient or when annealed up to $240^{\circ} \mathrm{C}$. In order to obtain a VCSEL with two dielectric DBRs, a low temperature metallic bonding process $\left(240^{\circ} \mathrm{C}\right)$ on silicon substrate has been used without damaging the optical properties of the device. The device optically pumped shows a laser emission at room temperature. 


\section{ACKNOWLEDGMENTS}

The authors wish to thank the Brittany region for supporting this project. 


\section{References}

Geske, J., K.G. Gan, Y.L. Okuno, J. Piprek and J.E. Bowers. IEEE J. Quantum. Electron. 40 1155, 2004.

Hubler, G. K., C.N. Waddell, W.G. Spitzer, J.E. Fredrickson, S.Prussin and R.G. Wilson. J. Appl. Phys. 50 3294, 1979.

Karim, A., J. Piprek, P. Abraham, D. Lofgreen, Y. Chiu and J. E. Bowers. IEEE J. Select. Topics Quantum Electron. 7 178, 2001.

Lee, C.C., C.Y. Wang, and G. Matijasevic, IEEE Trans. Comp. Hybrids. Manuf. Technol. 16, 311 (1993).

Levallois, C., A. Le Corre, S. Loualiche, O. Dehaese, H. Folliot, C. Paranthoen, F. Thoumyre, and C. Labbé, J. Appl. Phys. 98 043107, 2005.

Lin, H.C., W.H. Wang, K.C. Hsiek and K.Y. Cheng, Electron. Lett. 38 516, 2002.

Nakagawa, S., E. Hall, G. Almuneau, J.K. Kim, D.A. Buell, H. Kroemer and L.A. Coldren. IEEE J. Quantum. Electron. 7 224, 2001.

Pendry, J.B., and A. MacKinnon, Phys. Rev. Lett. 69 2772, 1992.

Piprek, J, H. Wenzel, H.J. Wünsche, D. Braun, and F. Henneberger, Proc. SPIE 2399 605, 1995.

Reddy, M.H.M., T. Asano, R. Koda, D.A. Buell and L.A. Coldren. IEEE Electron. Lett. 38 1181, 2002.

Richards, B.S., A. Lambertz, A.B. Sproul. Thin Solid Film 460 247, 2004.

Sun, D., W. Fan, P. Kner, J. Boucart, T. Kageyama, R. Pathak, D. Zhang and W. Yuen. IEEE Photon. Technol. Lett. 15 1677, 2003.

Syrbu, A., A. Mircea, A. Mereuta, A. Caliman, C.A. Berseth, G. Suruceanu, V. Iakovlev, M. Achtenhagen, A. Rudra and E. Kapon, IEEE Photon. Technol. Lett. 16 1230, 2004. 
Waddell, C.N., W.G. Spitzer, J.E. Fredrickson, G.K. Hubler and T.A. Kennedy, J. Appl. Phys. 55 4361, 1984. 


\section{Figure captions}

\section{Figure 1}

Refractive index and optical absorption versus nitrogen flow rate for a total pressure $\left(A r+N_{2}\right)$ maintained at $10^{-2}$ Torr. The circles show the experimental data about the refractive index and the solid curve is a guide for the eyes. The squares show the optical absorption measurement with their uncertainly of measurement.

\section{Figure 2}

Simulated and measured transmittance of 4.5 periods a-Si/a-SiN $\mathrm{x}_{\mathrm{x}} \mathrm{DBR}$ deposited on a glass substrate.

\section{Figure 3}

Simulated and measured reflectivity of a-Si/a-SiN ${ }_{x} \mathrm{DBR}$ of 4.5 periods deposited on a glass substrate under an incidence angle of $27^{\circ}$. The inset shows the details of the spectra between $1.5 \mu \mathrm{m}$ and $1.6 \mu \mathrm{m}$ wavelength.

\section{Figure 4}

Schematic diagram of the VCSEL fabrication process: (1) epitaxial growth - (2) bottom DBR deposition - (3) metallic layers deposition and contact between InP and Si substrate - (4) metallic bonding and InP substrate thinning - (5) InP substrate removal - (6) Deposition of the top DBR

\section{Figure 5}

Simulated and measured reflectivity of the VCSEL realized after the deposition of the top mirror. The inset shows the details of the spectra between $1.5 \mu \mathrm{m}$ and $1.56 \mu \mathrm{m}$ wavelength.

\section{Figure 6}

The reflectivity simulation of the fabricated VCSEL in normal incidence (thick and solid line). Photoluminescence measurement of $\mathrm{InGaAs} \mathrm{Q}_{1.18} \mathrm{QWs}$ on InP substrate (dashed 
line).Photoluminescence measurement of InGaAs/ $\mathrm{Q}_{1.18}$ QWs embedded in a microcavity (thin solid line).

\section{Figure 7}

Average power emitted by the VCSEL versus the peak power of the optical pump. The inset shows the laser spectrum near the threshold value. 
Figure 1

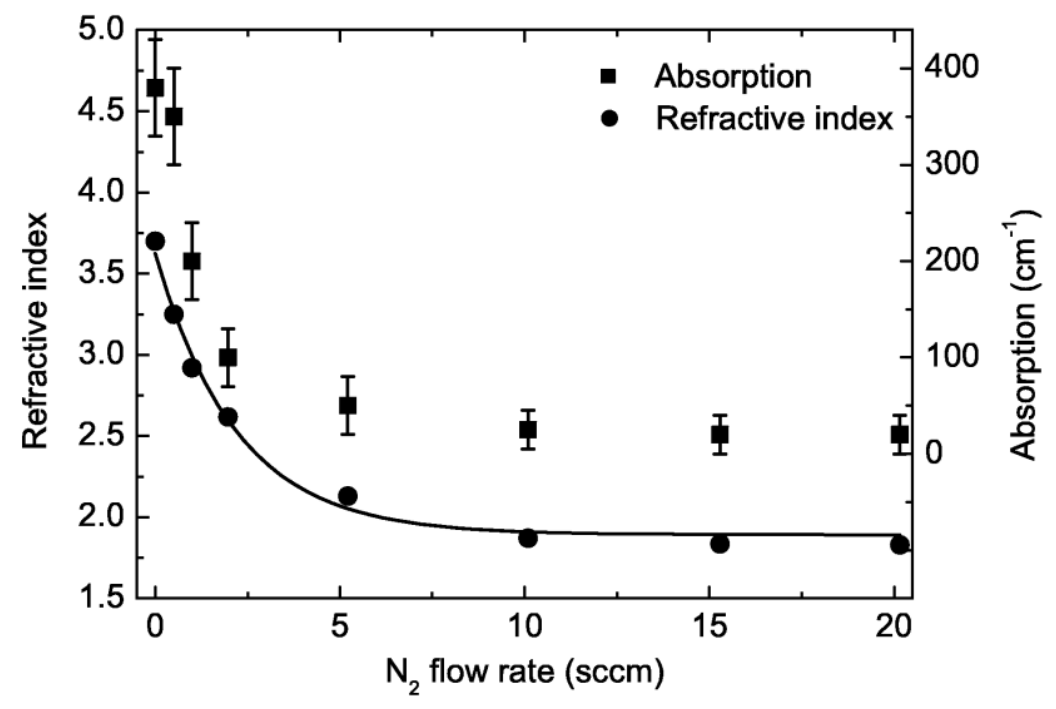


Figure 2

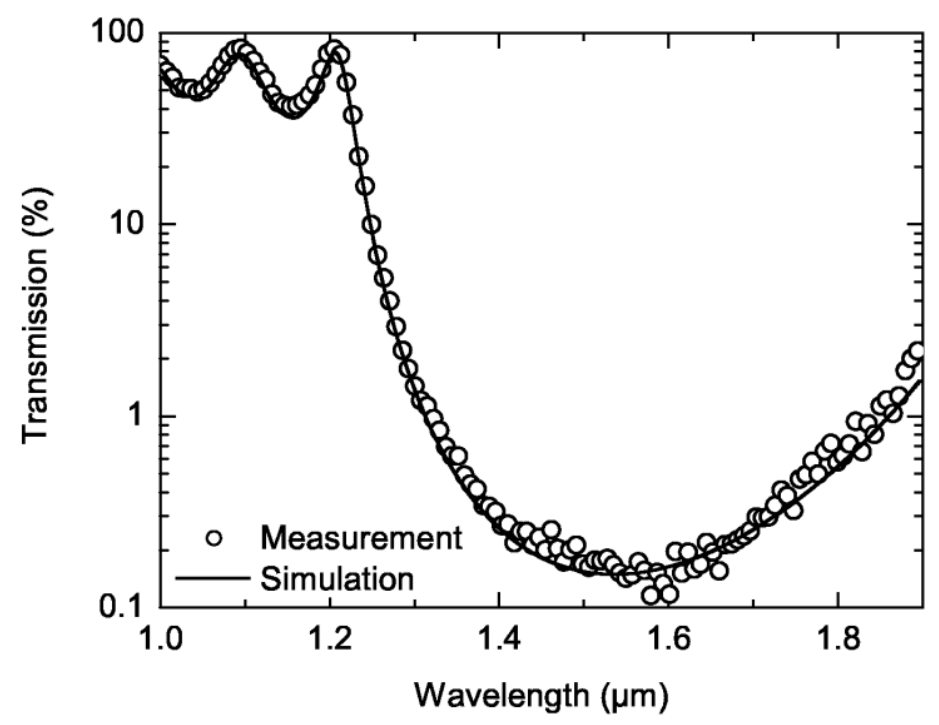


Figure 3

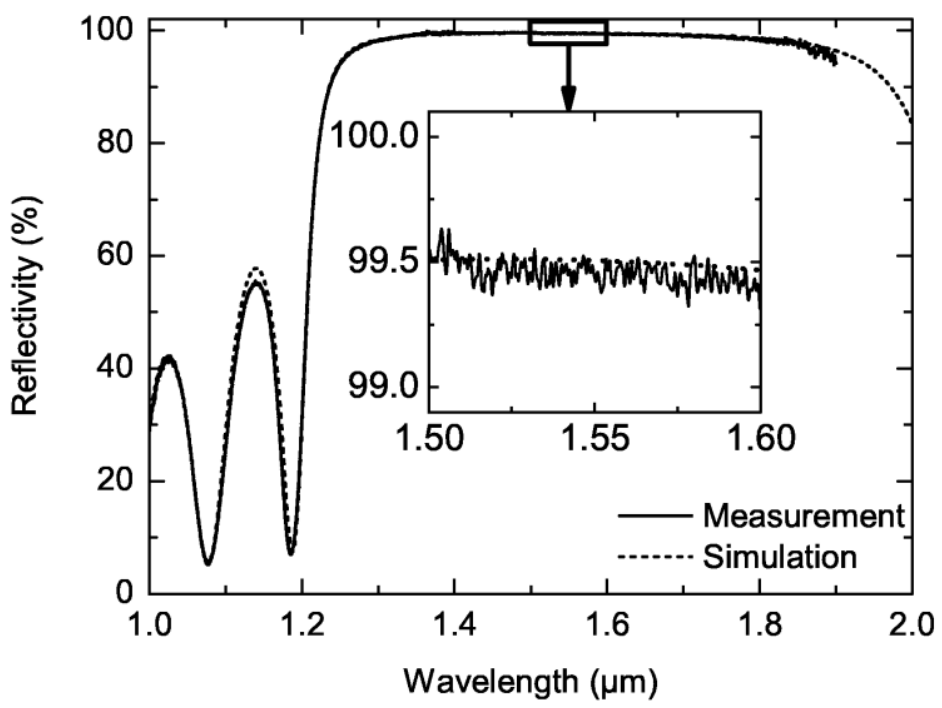


Figure 4

(1)

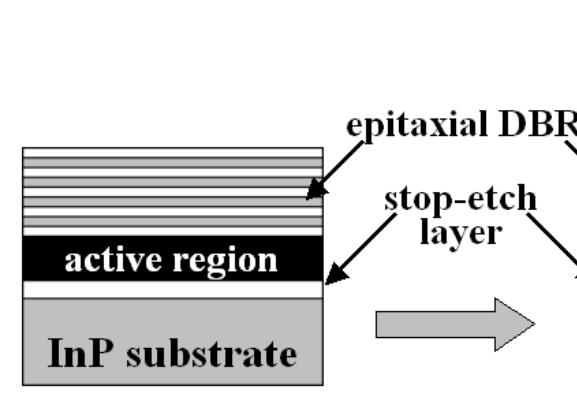

(6)

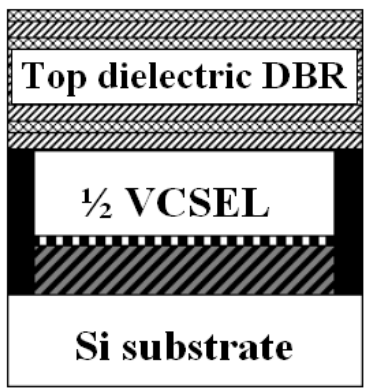

(2)

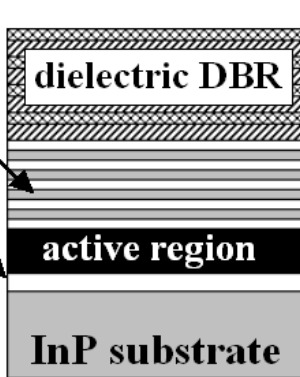

(5)

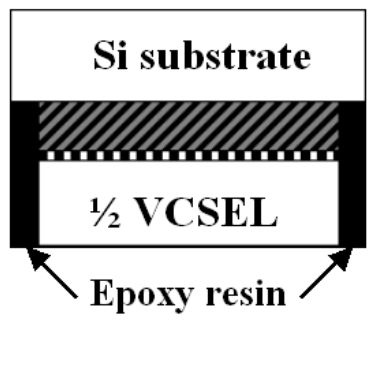

(3)
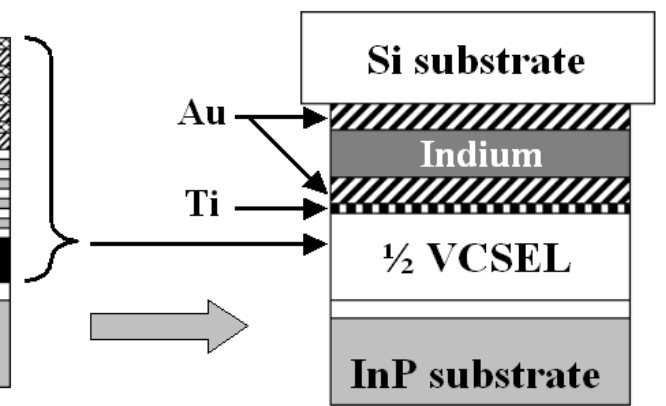

Temperature $240^{\circ} \mathrm{C}$

(4)

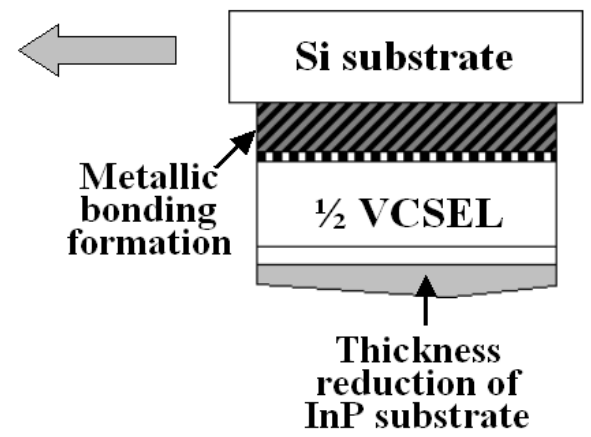


Figure 5

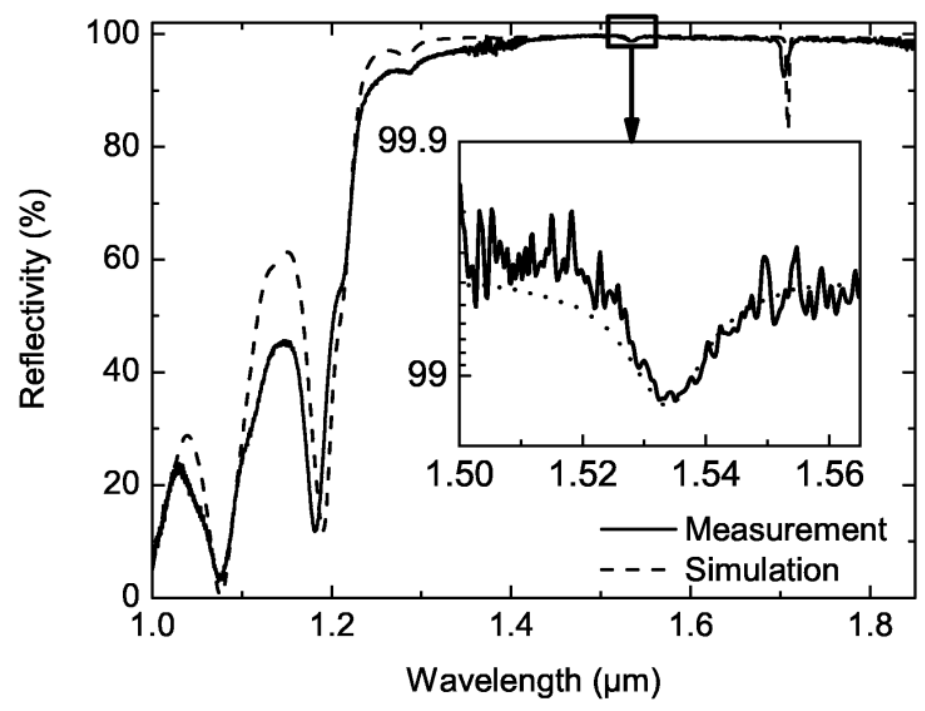


Figure 6

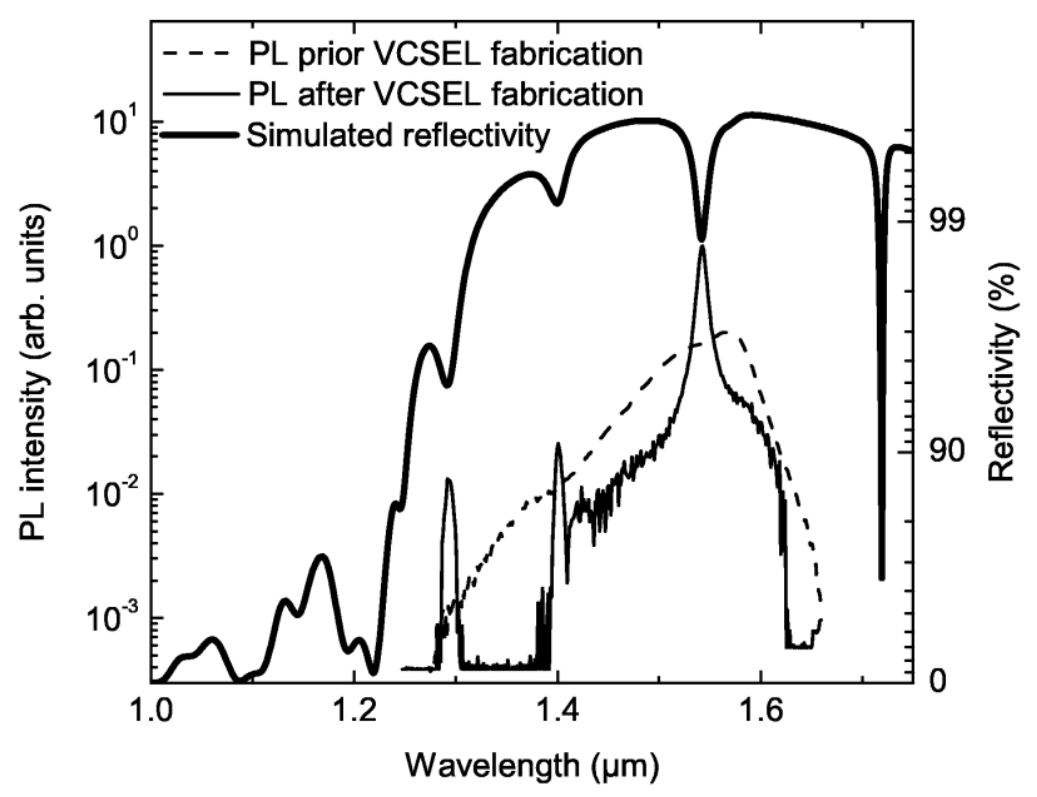


Figure 7

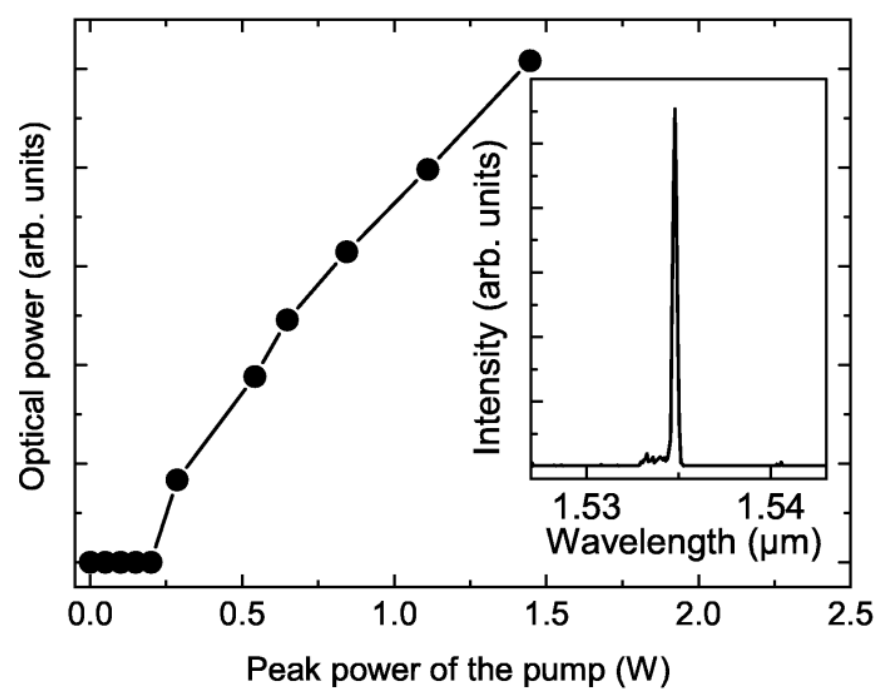

